\title{
Radiation dose optimization in pediatric temporal bone computed tomography: influence of tube tension on image contrast and image quality
}

\author{
Claude Bertrand Nauer • Christoph Zubler • \\ Christian Weisstanner • Christof Stieger • Pascal Senn • \\ Andreas Arnold
}

Received: 21 July 2011 /Accepted: 12 September 2011 /Published online: 23 September 2011

(C) Springer-Verlag 2011

\begin{abstract}
Introduction The purpose of this experimental study was to investigate the effect of tube tension reduction on image contrast and image quality in pediatric temporal bone computed tomography (CT).

Methods Seven lamb heads with infant-equivalent sizes were scanned repeatedly, using four tube tensions from 140 to $80 \mathrm{kV}$ while the CT-Dose Index (CTDI) was held constant. Scanning was repeated with four CTDI values from 30 to $3 \mathrm{mGy}$. Image contrast was calculated for the middle ear as the Hounsfield unit (HU) difference between bone and air and for the inner ear as the HU difference between bone and fluid. The influence of tube tension on high-contrast detail delineation was evaluated using a phantom. The subjective image quality of eight middle and inner ear structures was assessed using a 4-point scale (scores 1-2=insufficient; scores 3-4=sufficient).
\end{abstract}

C. B. Nauer $\cdot$ C. Zubler $\cdot$ C. Weisstanner

Institute of Diagnostic and Interventional Neuroradiology,

University Hospital Berne,

Berne, Switzerland

C. B. Nauer $(\bowtie)$

Zentrales Roentgeninstitut, Kantonsspital Graubünden, Loëstrasse 170

7000 Chur, Switzerland

e-mail: claudenauer@hotmail.com

C. Stieger $\cdot$ A. Arnold

Group for Artificial Hearing Research, ARTORG Center, University Berne,

Berne, Switzerland

P. Senn $\cdot$ A. Arnold

Department of ENT, Head and Neck Surgery,

University Hospital Berne,

Berne, Switzerland
Results Middle and inner ear contrast showed a near linear increase with tube tension reduction $(r=-0.94 /-0.88)$ and was highest at $80 \mathrm{kV}$. Tube tension had no influence on spatial resolution. Subjective image quality analysis showed significantly better scoring at lower tube tensions, with highest image quality at $80 \mathrm{kV}$. However, image quality improvement was most relevant for low-dose scans.

Conclusions Image contrast in the temporal bone is significantly higher at low tube tensions, leading to a better subjective image quality. Highest contrast and best quality were found at $80 \mathrm{kV}$. This image quality improvement might be utilized to further reduce the radiation dose in pediatric low-dose CT protocols.

Keywords Image quality - Temporal bone CT - Tube tension $\cdot$ Radiation exposure to patients $\cdot$ Pediatric CT

\section{Introduction}

Computed tomography (CT) is an important source of radiation exposure [1]. In recent years, a sharp increase in the use of this diagnostic modality has been observed [2]. As a consequence, there is growing concern over possible detrimental effects resulting from increasing radiation exposure, particularly in children [3, 4]. Children are more susceptible to the stochastic effects of radiation exposure than adults [3,4] and effective doses tend to be higher in children than in adults $[5,6]$. These facts emphasize the need for dose-reduction strategies in pediatric CT. Nevertheless, CT is a valuable diagnostic tool in pediatric radiology and neuroradiology, where it is mainly used in diagnostic work-up of the head, sinuses, and temporal bones. Temporal bone $\mathrm{CT}$ in children is used 
to assess the middle and inner ear, congenital hearing deficits, infection, and trauma [7-9].

In order to avoid excessive radiation exposure, pediatric protocols should be adapted to the size or age of the child [10-12]. Dose adaptation usually depends on reduction of the tube current-time product (milliampere seconds), though reduction of tube tension (kilovolts) has also been recommended $[5,12]$. The influence of the tube currenttime product on image quality is predictable as long as all other factors remain unchanged. The influence of tube tension on image quality is more complex. Generally, higher tissue absorption can be expected from lower beam energy. This effect is particularly effective for substances with high atomic numbers, like iodine, and is used in CT perfusion studies of the brain and in CT-angiography [13, 14]. Furthermore, there is evidence [5] that the contrast-toradiation dose ratio in objects with a small diameter (like an infant's head) is better at lower beam energies. Image quality in pediatric $\mathrm{CT}$ examinations may therefore benefit from the use of reduced tube tensions.

The aim of this study is

1. To verify whether tissue contrast in high-contrast objects, such as the temporal bone, is indeed higher at lower tube tensions

2. To quantify this effect

Fig. 1 Coronal section of the left sheep temporal bone. All scans were performed with a volumetric CT-Dose Index (CTDIvol) of $30 \mathrm{mGy}$. The eight structures used for the subjective image quality assessment are shown: a modiolus of cochlea and sharpness of fluid-air level in middle ear cavity, b spiral osseous lamina, $\mathbf{c}$ details of bony pattern in the mastoid bone and superior semicircular canal, d stapes head, e manubrium of malleus, and $\mathbf{f}$ oval window
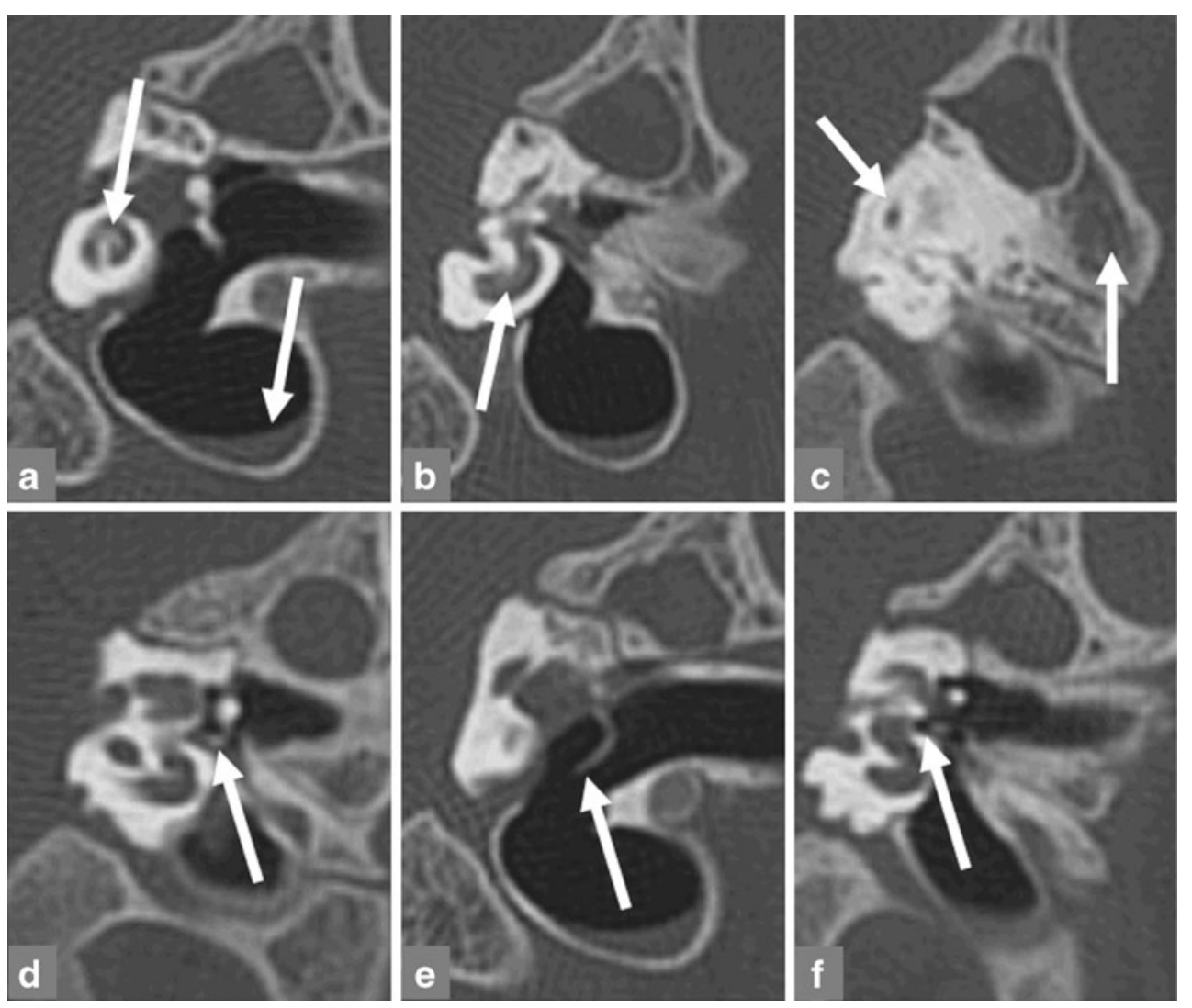
institution vs. mean $105 \times 125 \mathrm{~mm}$ in the lambs). Thus absorption properties of our lamb model were comparable to those of young children.

\section{Scanning technique}

All scans were performed with an eight-section CT scanner (Lightspeed Ultra, GE Healthcare, Milwaukee, WI) using a spiral scan with a pitch of 1 . Thin slices $(0.625 \mathrm{~mm})$ were reconstructed with an overlap of $68 \%$ in an edgeenhancing kernel ("Bone Plus"). A field of view of $170 \mathrm{~mm}$ was used, resulting in an estimated in-plane voxel size of $0.33 \mathrm{~mm}^{3}$.

For our experiment, the lamb heads were scanned with the four peak voltage settings available on our scanner $(140 / 120 / 100 / 80 \mathrm{kV})$ while the volumetric CT-Dose Index $\left(\mathrm{CTDI}_{\mathrm{vol}}\right.$, definition of the CTDI and details on dosimetry see below) was kept constant by adjusting the milliampere seconds. For example, $140 \mathrm{kV}$ and $80 \mathrm{mAs}$ resulted in a $\mathrm{CTDI}_{\mathrm{vol}}$ of $30 \mathrm{mGy}$, so do the combinations $120 \mathrm{kV} / 110 \mathrm{mAs}, 100 \mathrm{kV} / 170 \mathrm{mAs}$, or $80 \mathrm{kV} / 300 \mathrm{mAs}$. From the four scans obtained with equal $\mathrm{CTDI}_{\mathrm{vol}}$ but differing tube tensions, the isolated influence of tube tension on image quality could be evaluated. The effect of tube tension on image contrast is expected to be independent of the tube-current-time product, i.e., the $\mathrm{CTDI}_{\mathrm{vol}}$. However, the effect of better image contrast on image quality may be relevant, depending on the overall image quality level. The scan series described above was therefore performed with different $\mathrm{CTDI}_{\mathrm{vol}}$ values (30/15/7.5/3 mGy).

The CTDI is a measure of the radiation dose absorbed in the body, measured in milligray. It is obtained by measuring the radiation dose profile in a standard phantom at four points, three in the periphery $\left(\mathrm{CTDI}_{\mathrm{p}}\right)$, and one at the center $\left(\mathrm{CTDI}_{\mathrm{c}}\right)$. Then, the weighted CTDI is calculated as

$$
\mathrm{CTDI}_{\mathrm{w}}=1 / 3 \mathrm{CTDI}_{\mathrm{c}}+2 / 3 \mathrm{CTDI}_{\mathrm{p}} .
$$

For helical scanning, the $\mathrm{CTDI}_{\mathrm{vol}}$ is defined as $\mathrm{CTDI}_{\mathrm{vol}}=\mathrm{CTDI}_{\mathrm{w}} /$ pitch [17]. In modern CT scanners, the $\mathrm{CTDI}_{\mathrm{vol}}$ is indicated on the scanner panel. The accuracy of the dose reporting system had been tested earlier by personnel of the Federal Office of Public Health during a dose audit. The measurements were performed with a standard PMMA-phantom using a 100-mm pencil-shaped ionization chamber and a Barracuda electrometer (RTI Electronics, Mölndal, Sweden). The maximum difference between CTDI values indicated by the dose reporting system and the measured values was $11 \%$, with $3 \%$ for the low-dose range. According to our experience, only CTDI differences considerably greater than $10 \%$ are visible to the observer.
Tissue contrast and image noise measurements

To assess tissue contrast, absorption values (Hounsfield units, HU) were measured in the otic capsule, the vestibule, the malleus head and the aerated middle ear cavity by one reader $(\mathrm{CBN})$ on a $\mathrm{GE}$ Advantage Windows workstation, Version 4.1 (GE Healthcare, Milwaukee, WI). Because some of the evaluated structures were very small, a small region of interest (ROI) measuring only $1 \mathrm{~mm}^{2}$ had to be used. To compensate for the small ROI, eight measurements from neighboring areas were performed. The reader carefully avoided positioning the ROI at locations where partial volume effects might occur. Mean density was calculated from the 112 (14 ears $\times 8$ measurements) values obtained per $\mathrm{CTDI}_{\mathrm{vol}} /$ tube tension combination. Middle ear contrast was defined as the difference between the mean density of the malleus head and the mean density of air:

Contrast $_{\text {middle ear }}=\mathrm{HU}_{\text {malleus }}-\mathrm{HU}_{\text {air }}$

Inner ear contrast was defined as the difference between the mean density of the otic capsule and the vestibule:

Contrast $_{\text {inner ear }}=\mathrm{HU}_{\text {otic capsule }}-\mathrm{HU}_{\text {vestibule }}$

To assess image noise, measurements were obtained from the aerated middle ear. Image noise was defined as the first standard deviation of the HU measurement, as indicated by the software tool.

\section{Spatial resolution assessment}

To find a potential influence of tube tension on spatial resolution, a high-contrast phantom (QRM-3DSR, ARM, Moehrendorf, Germany) was scanned with the settings mentioned above, and the images were assessed by four readers blinded to the scanner settings. This phantom contains rows of drilled holes with diameters ranging from 4.00 to $0.4 \mathrm{~mm}$. Spatial resolution, measured in linepairs per centimeter $(\mathrm{lp} / \mathrm{cm})$ inversely correlates with the size of the smallest visible hole, see Fig. 2.

Subjective image quality assessment

Three experienced neuroradiologists (CBN 9 years, CZ 6 years, and $\mathrm{ChW} 5$ years of experience) rated the delineation of eight structures on the CT scans (see below in the text, see also Fig. 1) using a score ranging from 4 to 1 (4=excellent image quality, perfect delineation of structure; $3=$ acceptable image quality, structure assessable in all details; 2 =insufficient, structure is identifiable, but without visibility of details; and $1=$ structure cannot be identified). Four of the structures assessed represented bone versus soft 


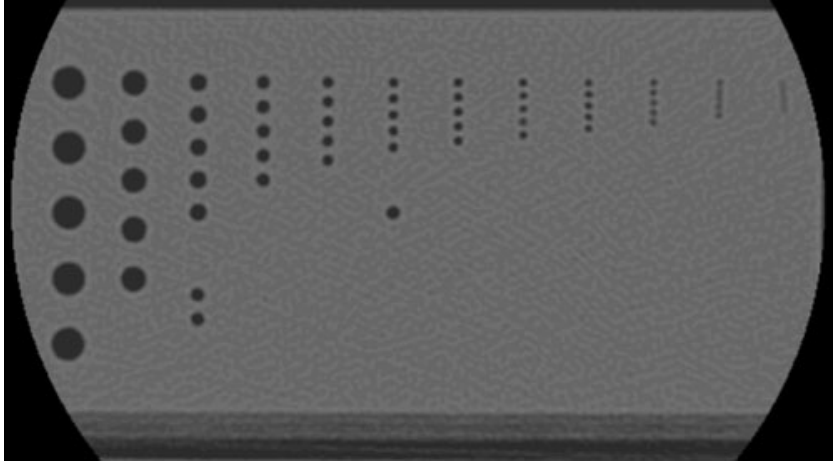

Fig. 2 CT scan of the high-contrast phantom used for spatial resolution assessment. Hole sizes ranges from $4 \mathrm{~mm}$ (left) to $0.4 \mathrm{~mm}$ (right). In this scan, holes are individually discernible down to $0.5 \mathrm{~mm}$ (second row from right), corresponding to a spatial resolution of ten line pairs per centimeter

tissue/fluid (The differentiation between soft tissue and fluid cannot be made from density or contrast on the scans reconstructed with an edge-enhancing kernel): spiral osseous lamina; modiolus of cochlea; superior semicircular canal; and trabecula in the unpneumatized mastoid. Three structures represented bone versus air contrast: oval window; stapes head/collum; malleus manubrium, and one represented air versus fluid contrast: border of air-fluid interface that was present in all middle ear cavities.

The scans were presented in random order and the readers were blinded to the scanning parameters. The reviews were performed on a workstation (Easy Vision, Philips Medical systems, Best, The Netherlands) that is also used in clinical work.

\section{Statistical analysis}

Pearson's correlation coefficient $r$ was calculated to find a potential correlation between tube tension and tissue contrast/image noise.

The tissue contrast data groups were analyzed with an ANOVA test to find significant differences across data groups, $p<0.05$ was considered significant. Subsequently, data were compared pairwise using the Bonferroni post test.

The image quality ratings for the anatomical structures and the three reviewers were pooled for each $\mathrm{CTDI}_{\mathrm{vol}} / \mathrm{kV}$ value and were then analyzed for differences between the tube tension groups using the Friedman test. Again, $p<0.05$ was considered significant. Further, the data were analyzed groupwise for significant differences between the tube tension groups 140/120,140/100,140/80,120/100, 120/80, and $100 / 80 \mathrm{kV}$ with Dunn's post test $(p<0.05$ was considered significant).

The difference between image quality score 4 (excellent) and 3 (acceptable) is not as important clinically as the difference between 3 (acceptable) and 2 (insufficient). Hence, to better assess the clinical significance of potential image quality differences, the scores were additionally dichotomized (1, 2=insufficient, 3, 4=sufficient image quality) and analyzed thereafter.

\section{Results}

Image contrast measurements

The tissue density data are shown in Fig. 3. Mean values increased when tube tension was reduced. The relative increase, however, varied across structures. While the relative density increase was only $+3 \%$ for air when tube tension was reduced from 140 to $80 \mathrm{kV}$, it was $+45-50 \%$ for the osseous structures and $+61 \%$ for the vestibule. The absolute density increase in $\mathrm{HU}$ from 140 to $80 \mathrm{kV}$ was about ten times higher for the otic capsule than for the vestibule and about 30 times higher for the malleus than for air. As a consequence, the contrast between tissues was higher at lower tube tensions, and contrast and tube tension for both the inner and the middle ear correlated well inversely ( $r=-0.94 /-0.88$; Fig. 4$)$. These contrast differences were highly significant for all pairwise comparisons for both the middle and the inner ear.

Image noise measurements

The mean image noise was $79.5 \mathrm{HU}$ at $140 \mathrm{kV}, 83.5 \mathrm{HU}$ at $120 \mathrm{kV}, 95.1 \mathrm{HU}$ at $100 \mathrm{kV}$, and 102.9 at $80 \mathrm{kV}(r=-0.99)$.

Spatial resolution

No correlation between tube tension and spatial resolution was found. The mean spatial resolution was $8.3 \mathrm{lp} / \mathrm{cm}$ at $140 \mathrm{kV}, 8.6 \mathrm{lp} / \mathrm{cm}$ at $120 \mathrm{kV}, 8.4 \mathrm{lp} / \mathrm{cm}$ at $100 \mathrm{kV}$, and $8.2 \mathrm{lp} /$ $\mathrm{cm}$ at $80 \mathrm{kV}$.

Subjective image quality assessment

The frequencies of the subjective image quality scores are shown in Fig. 5a-d.

For the three higher doses $\left(\mathrm{CTDI}_{\mathrm{vol}} 30 / 15 / 7.5 \mathrm{mGy}\right)$, a trend towards a higher frequency of score 4 was noted for the images obtained with lower tube tensions, while the scores 3 and 2 were given less often. For the lowest dose (3 mGy), scores 2, 3, and 4 were selected with similar frequencies for tube tensions $140-100 \mathrm{kV}$, but scores 3 and 4 were more frequently chosen at $80 \mathrm{kV}$ and score 2 was much less frequent. The frequency of score 1 was very low across scans. Statistical analysis revealed overall differences between the $\mathrm{kV}$-groups to be significant 
Fig. 3 Box-whisker plots of middle and inner ear density values (Hounsfield units) versus tube tension (kilovolts), a otic capsule, b vestibule, c malleus, and $\mathbf{d}$ air. The whiskers indicate 5-95 percentiles

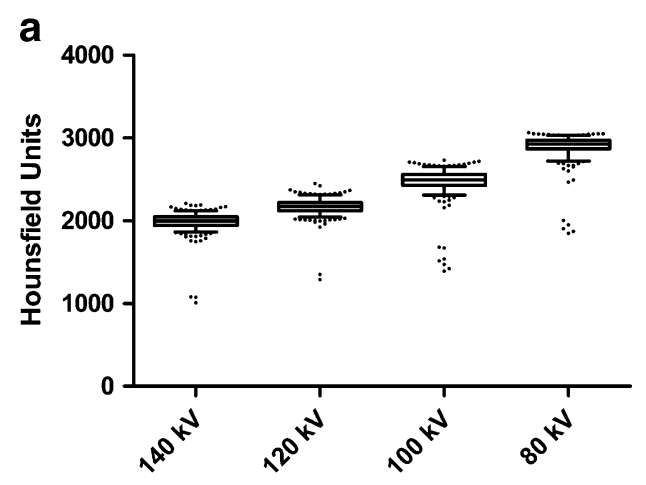

b

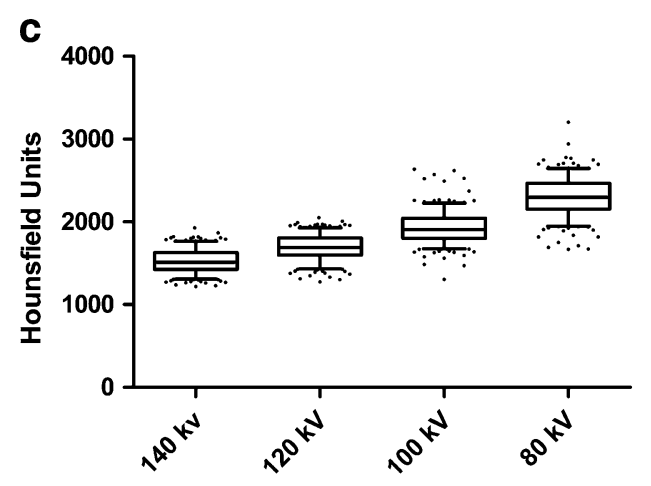



d

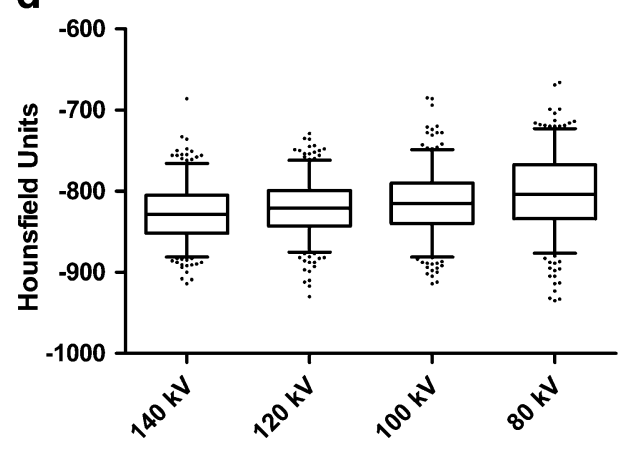

(Friedman, $p<0.05$ ). Pairwise analysis of the data columns with Dunn's post test showed significant differences for all comparisons with a voltage difference of $40 \mathrm{kV}$ (e.g., 120 vs. $80 \mathrm{kV}$ ), while 140 vs. $120 \mathrm{kV}$ and 120 vs. $100 \mathrm{kV}$ were not significantly different, however 100 vs. $80 \mathrm{kV}$ was significantly different as well.

The dichotomized data are shown in Fig. 6. For the sake of clarity, the relative frequencies of the sufficient scores are shown as percentages. We found a trend toward a higher frequency of sufficient image quality scores with an $80-\mathrm{kV}$ tube tension for all radiation doses, but there was a clear advantage of using $80 \mathrm{kV}$ for the two lowest doses (7.5/ $3 \mathrm{mGy}$ ) only. Overall differences between the $\mathrm{kV}$-groups were significant, but the pairwise comparison only showed statistically significant differences for $140 / 80 \mathrm{kV}$ at $30 \mathrm{mGy}$ and $140 / 80 \mathrm{kV}$ at $7.5 \mathrm{mGy}$. At $3 \mathrm{mGy}$, significant differences were found for pairs 140/80, 120/80, and $100 / 80 \mathrm{kV}$. The comparison between 120 and $80 \mathrm{kV}$ is the clinically most relevant comparison. There was no or only a marginal score increase between 120 - and $80-\mathrm{kV}$ tube tensions at the two highest radiation doses. However, the increase was $10.5 \%$ at $7.5 \mathrm{mGy}$ and $19.8 \%$ at $3 \mathrm{mGy}$.

\section{Discussion}

The aim of this experimental study was to test to what extent image contrast and image quality in pediatric temporal bone $\mathrm{CT}$ can be improved at a given radiation exposure level by use of a lower tube tension. Any way to improve image quality without increasing radiation exposure would be beneficial. Moreover, a better image quality could be used for diagnostic purposes ("see more") or for a further radiation dose reduction ("do less harm").
Fig. 4 Box-whisker plots of middle (a) and inner ear (b) magnitude of image contrast (Hounsfield units) versus tube tension (kilovolts)
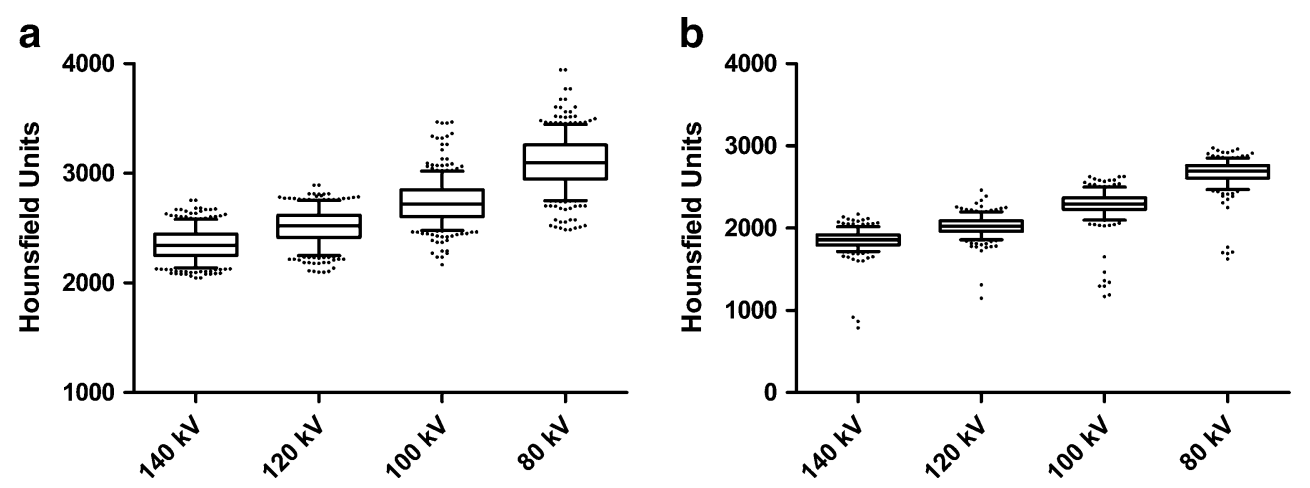
a

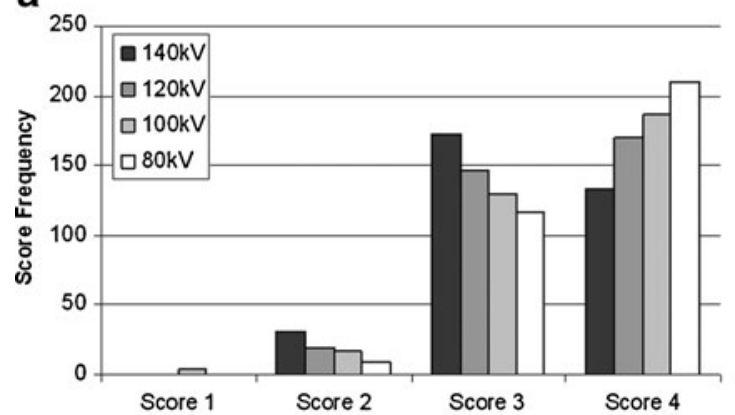

C

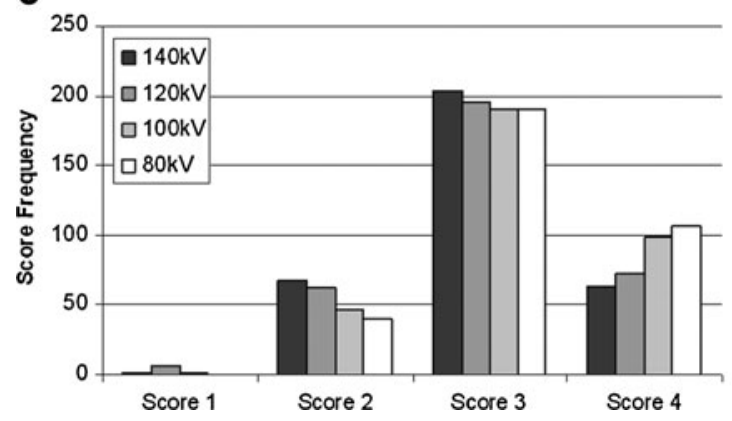

Fig. 5 Frequencies of subjective image quality scores 1-4 relative to tube tension (kilovolts), at different radiation doses; a $30 \mathrm{mGy}$, b $15 \mathrm{mGy}, \mathbf{c} 7.5 \mathrm{mGy}$, and d $3 \mathrm{mGy}$. Image quality tends to be higher at

Using lamb heads as a model for young children, we found significantly higher image contrast for middle and inner ear structures at lower tube tensions. For example, the tissue contrast between the ossicular chain and the surrounding air increased more than $500 \mathrm{HU}$ when the tube tension was reduced from 120 to $80 \mathrm{kV}$. We found, on the other hand, higher image noise at lower tube tensions. In light of these two contradicting influences on image quality, it was necessary to assess whether tube tension reduction influences overall image quality in addition to image contrast. This step was even more important because scientific and clinical assessments of image quality do not necessarily agree [18].

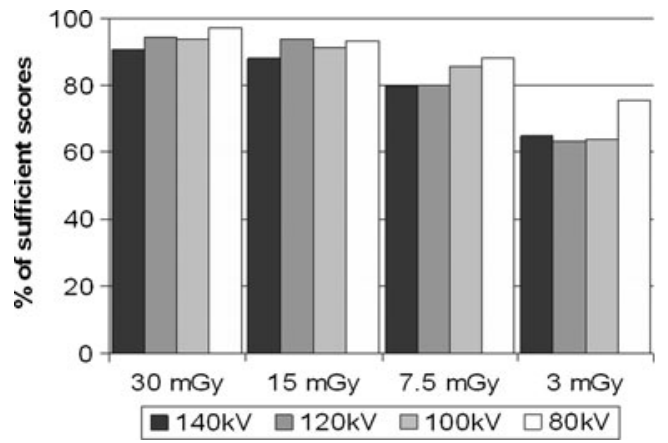

Fig. 6 Frequency of the (dichotomized) sufficient image quality scores as percentages b

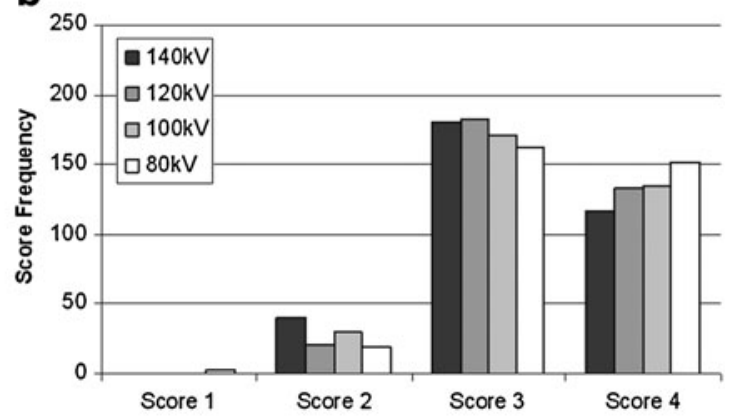

d

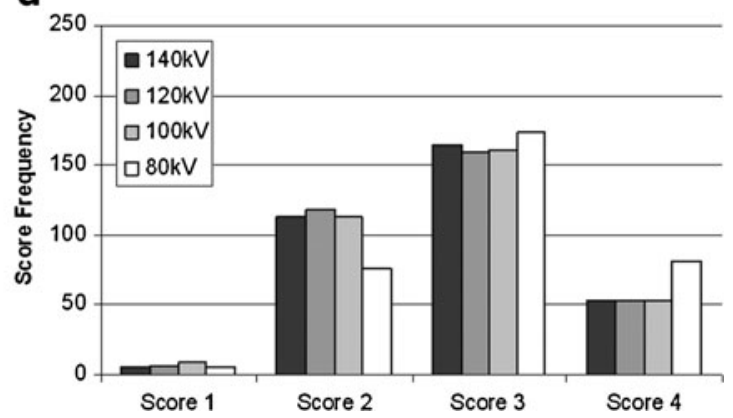

lower kilovolts for all doses, with score 4 gradually becoming more frequent with stepwise tube tension reduction while scores 2 and 3 become less frequent

Subjective image quality gradually improved in our experimental setting when tube tension was lowered, with an optimum image quality at $80 \mathrm{kV}$, the lowest tube tension value assessed in our study. The statistical analysis showed significant differences for data groups where the tube tension difference was $40 \mathrm{kV}$ (for example 120 vs. $80 \mathrm{kV}$ ). Because in clinical routine most CT scans are performed with $120 \mathrm{kV}$, reducing tube tension to $80 \mathrm{kV}$ has hence the potential to significantly improve image quality.

When interpreting image quality ratings, it is important to consider that the impact of image quality improvement on the radiologist's needs is not the same when image quality improves from acceptable (image quality score 3 ) to excellent (score 4) as when it increases from insufficient (score 2) to acceptable (score 3). Thus, we analyzed our data with a dichotomized scoring system (sufficient vs. insufficient). This way of looking at our data showed a statistically significant and potentially clinically relevant image quality gain for $80 \mathrm{kV}$ relative to $120 \mathrm{kV}$ for the two lowest radiation doses. Children below 2 years of age are scanned at our institution with a low-dose protocol using a $\mathrm{CTDI}_{\mathrm{vol}}$ of $9 \mathrm{mGy}$. At $7.5 \mathrm{mGy}$, which is in the range of our clinical protocol, $80 \mathrm{kV}$ still showed a clear advantage over $120 \mathrm{kV}$. The use of $80 \mathrm{kV}$ instead of $120 \mathrm{kV}$ could therefore improve image quality significantly in a clinically applied low-dose protocol, and this image quality gain might be 

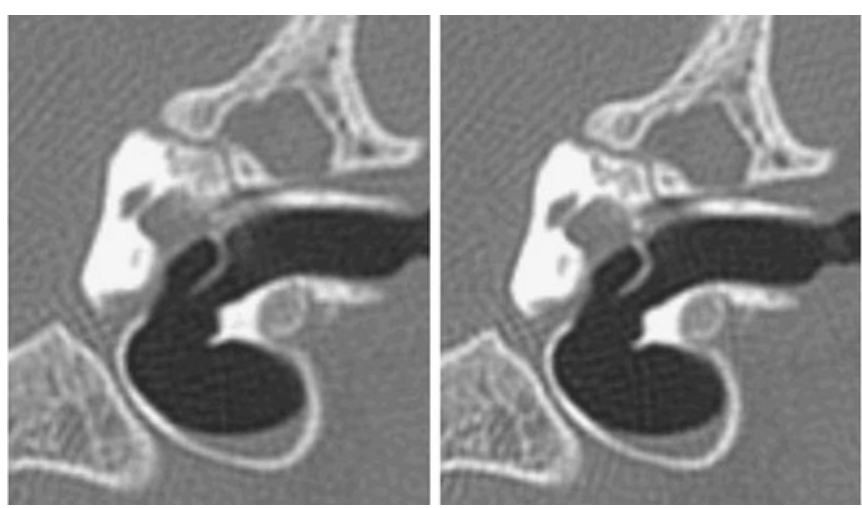

Fig. 7 Scans of a lamb temporal bone performed with $30 \mathrm{mGy}$ and tube tension from $140 \mathrm{kV}$ (left) to $80 \mathrm{kV}$ (right). Note the improved contrast between the malleus handle and the air in the middle ear

used to obtain a further dose reduction. At higher doses however, when overall image quality is good, $80 \mathrm{kV}$ does not offer a real advantage over $120 \mathrm{kV}$ even if the image contrast is higher (see Figs. 7 and 8).

With the advent of digital volume tomography (DVT), a low-dose alternative offering even a higher spatial resolution than $\mathrm{CT}$ has become available for the temporal bone [19], and it may not seem prudent to scan patients with CT anymore. However, patients must usually be positioned upright in the DVT for the scanning process, and therefore DVT is not suited for very young patients. Additionally, DVT is not universally available. Optimization of low-dose CT protocols of the temporal bone in children is therefore still relevant.

Our animal model was chosen because of the anatomical and physical similarities to children below 2 years of age. We did not, however, assess the upper limit of age at which lower tube tension renders a better image quality for a given $\mathrm{CTDI}_{\mathrm{vol}}$. The thicker bony structures resulting from growing head size and calvarial thickness in older children should cause more beam-hardening effects, resulting in a
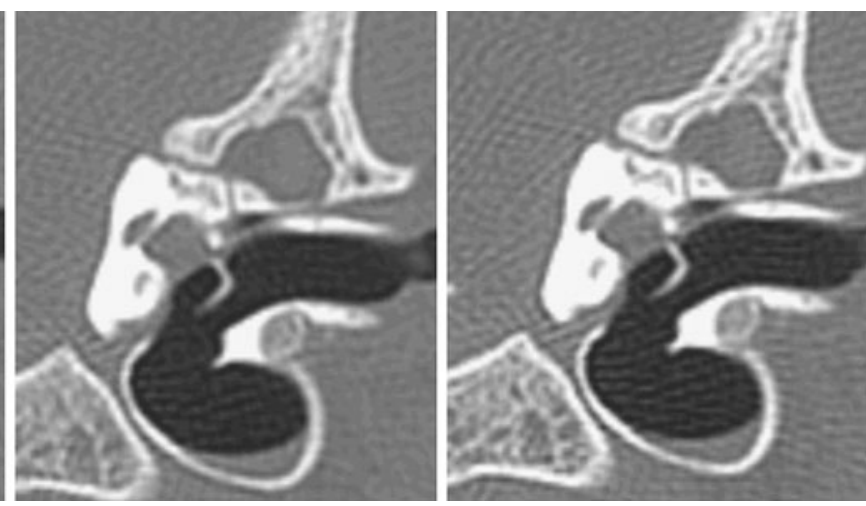

cavity at the lowest tube tension. Noise is slightly higher at $80 \mathrm{kV}$. All images are shown with identical window/level settings

higher beam energy at the detector [5]. This could impair the image contrast improvement we found. However, while head size increases rapidly until the age of 2 , the rate of growth slows down, becoming more modest until the age of 18 years [20]. Thus, a significant image quality improvement is conceivable well beyond the age of 2 years, with the effect gradually becoming less pronounced with advancing age. In adolescent and adult heads, photon-starving effects may pose a problem that limits the use of low tube tension.

\section{Conclusion}

With this experimental study we confirm that image contrast in temporal bone $\mathrm{CT}$ is higher at lower tube tension. We found a nearly linear inverse correlation between image contrast and tube tension. This higher contrast leads to a better image quality as subjectively perceived by the radiologist, with highest image contrast and image quality at $80 \mathrm{kV}$. The magnitude of image quality improvement is marginal for high-dose, high-quality scans,
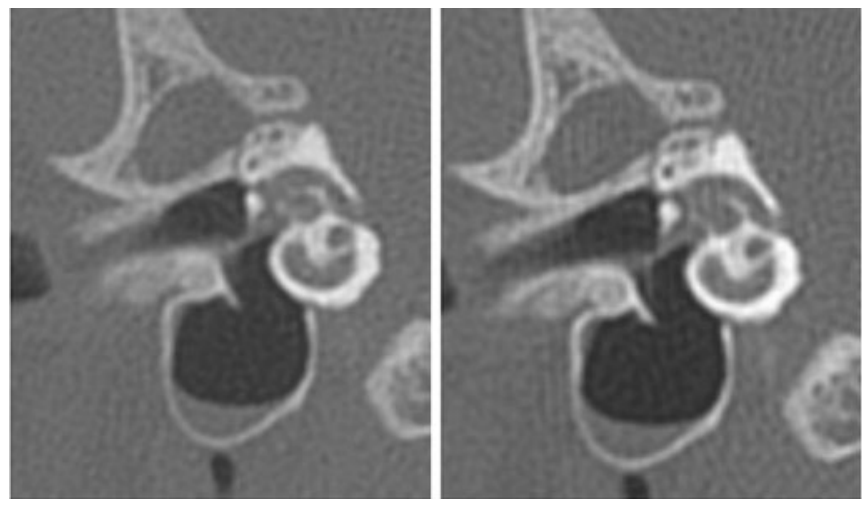

Fig. 8 Scans of a lamb temporal bone performed with $7 \mathrm{mGy}$, a dose corresponding to a clinical low-dose protocol. Tube tensions range from $140 \mathrm{kV}$ (left) to $80 \mathrm{kV}$ (right). Note the better delineation of the
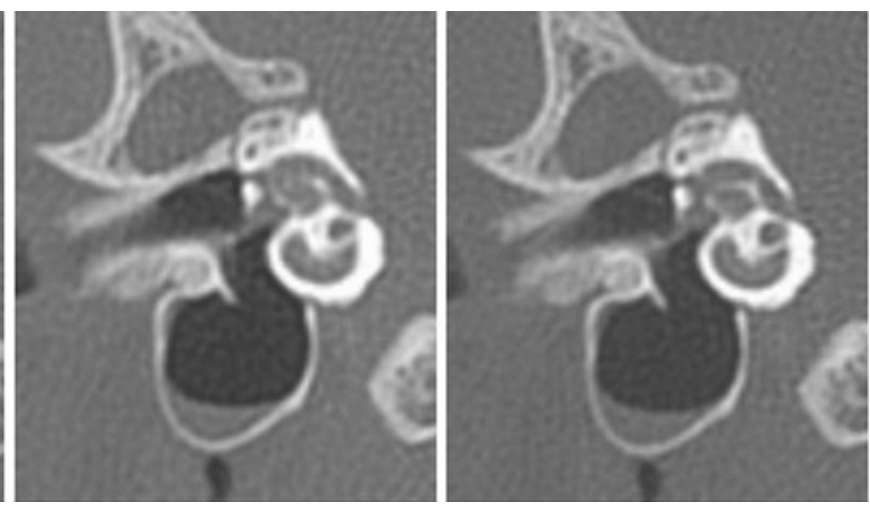

spiral osseous lamina at the lowest tube tension. Osseous structures like the otic capsule show higher density at low beam energy 
but seems to be clinically relevant for scans with a limited overall image quality, i.e., for low-dose scans. The use of $80 \mathrm{kV}$ might hence be useful in pediatric low-dose CT protocols, and allow for a further dose reduction.

Acknowledgments The authors thank M. Jonczy, N. Paquier, and P. Flach for their participation in the image quality assessment of the high-contrast phantom.

Conflict of interest We declare that we have no conflict of interest.

\section{References}

1. Fazel R, Krumholz HM, Wang Y, Ross JS, Chen J, Ting HH, Shah ND, Nasir K, Einstein AJ, Nallamothu BK (2009) Exposure to low-dose ionizing radiation from medical imaging procedures. N Engl J Med 361(9):849-857. doi:10.1056/NEJMoa0901249

2. Brenner DJ, Hall EJ (2007) Computed tomography - an increasing source of radiation exposure. N Engl J Med 357(22):2277-2284. doi:10.1056/NEJMra072149

3. Linet MS, Kim KP, Rajaraman P (2009) Children's exposure to diagnostic medical radiation and cancer risk: epidemiologic and dosimetric considerations. Pediatr Radiol 39(Suppl 1):S4-S26. doi:10.1007/s00247-008-1026-3

4. Brenner D, Elliston C, Hall E, Berdon W (2001) Estimated risks of radiation-induced fatal cancer from pediatric CT. AJR Am J Roentgenol 176(2):289-296

5. Huda W, Lieberman KA, Chang J, Roskopf ML (2004) Patient size and X-ray technique factors in head computed tomography examinations. II. Image quality. Med Phys 31(3):595-601

6. Khursheed A, Hillier MC, Shrimpton PC, Wall BF (2002) Influence of patient age on normalized effective doses calculated for CT examinations. Br J Radiol 75(898):819-830

7. Bettman R, Beek E, Van Olphen A, Zonneveld F, Huizing E (2004) MRI versus CT in assessment of cochlear patency in cochlear implant candidates. Acta Otolaryngol 124(5):577581

8. Robson CD (2006) Congenital hearing impairment. Pediatr Radiol 36(4):309-324. doi:10.1007/s00247-005-0042-9
9. Vazquez E, Castellote A, Piqueras J, Mauleon S, Creixell S, Pumarola F, Figueras C, Carreno JC, Lucaya J (2003) Imaging of complications of acute mastoiditis in children. Radiographics 23 (2):359-372

10. Radiation risks and pediatric computed tomography (CT): a guide for health care providers National Cancer Institute Website. http://www.cancer.gov/cancertopics/causes/radiation/radiation-risks-pediatric-CT. Accessed 13th of July 2011

11. Goske MJ, Applegate KE, Boylan J, Butler PF, Callahan MJ, Coley BD, Farley S, Frush DP, Hernanz-Schulman M, Jaramillo D, Johnson ND, Kaste SC, Morrison G, Strauss KJ, Tuggle N (2008) The Image Gently campaign: working together to change practice. AJR Am J Roentgenol 190(2):273-274. doi:10.2214/ AJR.07.3526

12. Vock P (2005) CT dose reduction in children. Eur Radiol 15 (11):2330-2340. doi:10.1007/s00330-005-2856-0

13. Szucs-Farkas Z, Semadeni M, Bensler S, Patak MA, von Allmen G, Vock P, Schindera ST (2009) Endoleak detection with CT angiography in an abdominal aortic aneurysm phantom: effect of tube energy, simulated patient size, and physical properties of endoleaks. Radiology 251(2):590-598. doi:10.1148/radiol.2512081687

14. Wintermark M, Maeder P, Verdun FR, Thiran JP, Valley JF, Schnyder P, Meuli R (2000) Using $80 \mathrm{kVp}$ versus $120 \mathrm{kVp}$ in perfusion $\mathrm{CT}$ measurement of regional cerebral blood flow. AJNR Am J Neuroradiol 21(10):1881-1884

15. Seibel VA, Lavinsky L, De Oliveira JA (2006) Morphometric study of the external and middle ear anatomy in sheep: a possible model for ear experiments. Clin Anat 19(6):503-509. doi: $10.1002 /$ ca.20218

16. Seibel VA, Lavinsky L, Irion K (2006) CT-Scan sheep and human inner ear morphometric comparison. Braz J Otorhinolaryngol 72 (3):370-376

17. Suetens P (2009) Fundamentals of medical imaging, 2nd edn. Cambridge University Press, Cambridge

18. Walsh C, Dowling A, Meade A, Malone J (2005) Subjective and objective measures of image quality in digital fluoroscopy. Radiat Prot Dosim 117(1-3):34-37. doi:10.1093/rpd/nci708

19. Miracle AC, Mukherji SK (2009) Conebeam CT of the head and neck, part 2: clinical applications. AJNR Am J Neuroradiol 30 (7):1285-1292. doi:10.3174/ajnr.A1654

20. Huda W, Lieberman KA, Chang J, Roskopf ML (2004) Patient size and $\mathrm{x}$-ray technique factors in head computed tomography examinations. I. Radiation doses. Med Phys 31(3):588-594 